\title{
Pharmacogenetic testing through the direct-to-consumer genetic testing company 23andMe
}

\author{
Mengfei Lu', Cathryn M. Lewis ${ }^{1,2}$ and Matthew Traylor ${ }^{1 *}$ (D)
}

\begin{abstract}
Background: Rapid advances in scientific research have led to an increase in public awareness of genetic testing and pharmacogenetics. Direct-to-consumer (DTC) genetic testing companies, such as $23 a n d M e$, allow consumers to access their genetic information directly through an online service without the involvement of healthcare professionals. Here, we evaluate the clinical relevance of pharmacogenetic tests reported by 23andMe in their UK tests.
\end{abstract}

Methods: The research papers listed under each 23andMe report were evaluated, extracting information on effect size, sample size and ethnicity. A wider literature search was performed to provide a fuller assessment of the pharmacogenetic test and variants were matched to FDA recommendations. Additional evidence from CPIC guidelines, PharmGKB, and Dutch Pharmacogenetics Working Group was reviewed to determine current clinical practice. The value of the tests across ethnic groups was determined, including information on linkage disequilibrium between the tested SNP and causal pharmacogenetic variant, where relevant.

Results: 23andMe offers 12 pharmacogenetic tests to their UK customers, some of which are in standard clinical practice, and others which are less widely applied. The clinical validity and clinical utility varies extensively between tests. The variants tested are likely to have different degrees of sensitivity due to different risk allele frequencies and linkage disequilibrium patterns across populations. The clinical relevance depends on the ethnicity of the individual and variability of pharmacogenetic markers. Further research is required to determine causal variants and provide more complete assessment of drug response and side effects.

Conclusion: 23andMe reports provide some useful pharmacogenetics information, mirroring clinical tests that are in standard use. Other tests are unspecific, providing limited guidance and may not be useful for patients without professional interpretation. Nevertheless, DTC companies like 23andMe act as a powerful intermediate step to integrate pharmacogenetic testing into clinical practice.

\section{Background}

Recent advances in technology have enabled comprehensive characterization of the genetic component underlying many complex diseases, traits, and responses to medication. This new information has enabled genetic testing to become more widely available in healthcare, and can be used to assess risk of inherited conditions and predict response to medication. Such testing has the potential to reduce drug-related adverse events, as well as to increase

\footnotetext{
* Correspondence: mt628@medschl.cam.ac.uk

${ }^{1}$ Department of Medical and Molecular Genetics, King's College London, 8th Floor Tower Wing, Guy's Hospital, Great Maze Pond, London SE1 9RT, UK Full list of author information is available at the end of the article
}

the effectiveness of drugs by assessing how sensitive an individual might be to a given pharmaceutical. Several pharmacogenetic tests have become standard clinical practice and others are supported by strong research evidence. However, challenges exist in moving pharmacogenetic testing from a research endeavor to point-of-care implementation.

Traditionally, genetic tests have been ordered in clinical settings but direct-to-consumer (DTC) genetic testing companies allow consumers to access their genetic information through an online service without the intermediary of a healthcare professional. A 2012 review of online companies offering pharmacogenetic testing identified 
eight companies providing at least one such test, either DTC or through a healthcare practitioner [1]. 23andMe was the most comprehensive DTC pharmacogenetic testing company, and it remains active, although its portfolio of pharmacogenetic tests has reduced substantially since scrutiny by the FDA in 2013.

Pharmacogenetic testing is an interesting market for DTC companies, since test results only become actionable when a relevant drug is prescribed, which may be at the point of testing or many years later. This contrasts with genetic testing for disease variants where the increased or decreased - risk is of immediate relevance for the participant. It may be cost-effective for individuals to undergo pharmacogenetic testing once in their adult life, and store the results, informing physicians whenever a new drug is prescribed so any relevant gene-drug associations may be checked. Such pre-emptive pharmacogenetic testing may become routine practice, with data stored in the electronic health record (EHR), but there are few current implementations of this [2]. The introduction of DTC genetic testing has raised significant controversy amongst clinicians, academic researchers and the general public [3]. One of the major concerns is the potential for misunderstanding and misinterpretation of the test results, particularly when pre- or post-test genetic counselling is not provided.

Here we study the pharmacogenetic tests offered by 23andMe to UK customers. 23andMe began offering health-related tests to a UK market in December 2014, when their US tests covered only ancestry testing, following FDA concerns over their health tests. Currently, 23andMe provides reports on over 100 health conditions and traits for UK customers. These reports include (1) recessive inherited variants for conditions such as cystic fibrosis and beta-thalassaemia, (2) dominantly inherited genetic risk factors like variants in the $B R C A 1$ and BRCA2 genes, (3) drug response tests like abacavir hypersensitivity and warfarin sensitivity and (3) prediction of traits such as hair colour and earwax type. 23andMe genotypes consumer's DNA on the Illumina HumanOmniExpress-24 format chip, with more than 715,000 single nucleotide polymorphisms (SNPs), together with custom content specific for their tests (https://www.23andme.com/en-gb/health/; accessed 3rd November, 2016). Consumer reports list each relevant genotype together with a summary, and further information on the test. 23andMe's criteria [4] for including a genetic test in its portfolio are that the drug response tests are eligible if there are either existing clinical practice guidelines provided by the Clinical Pharmacogenetics Implementation Consortium (CPIC) and other clinical organisations, or if information from regulatory agencies or in drug labels "acknowledges the impact of the genetic marker on drug response". 23andMe regards pharmacogenetic tests as potentially eligible if "there is meaningful interpretation of a positive result", and at least three scientific research papers identify consistent clinical effects of the marker tested [4].

23andMe currently provides reports on 12 pharmacogenetic tests for UK customers. Here, we evaluate the extent to which these tests 1) represent the latest scientific and clinical literature, 2) reflect recommendations from the U.S. FDA and CPIC, and 3) are applicable across different ethnic groups.

\section{Methods}

\section{Data sources and search strategy}

Each test within the Drug Response section of 23andMe UK genetic testing report was reviewed (https://www.23andme.com/en-gb/health/reports). For all 12 pharmacogenetic tests, the 23andMe website provides details of 1) the drug, disorder and variant(s) relevant for the pharmacogenetic test, 2) possible outcomes by genotype, 3) interpretation of test results, and 4) academic papers reporting the pharmacogenetic association. Full technical information on the SNPs used to determine test results are given, enabling each test to be assessed fully.

The research papers listed under each 23andMe report were evaluated, extracting information on effect size, sample size and ethnicity. A wider literature search was performed using PubMed and EMBASE assess the pharmacogenetic test fully. The variants reported by 23andMe were matched to the FDA Table of Pharmacogenomic Biomarkers in Drug Labelling [5]. Additional evidence from CPIC guidelines, [6] PharmGKB, [7] and Dutch Pharmacogenetics Working Group (DPWG) [8] was reviewed to determine current clinical practice. SNP allele frequencies and linkage disequilbrium (LD) between SNPs were determined using the 1000 genomes project data through SNAP (http://archive.broadinstitu te.org/mpg/snap/) and LDlink (https://analysistools.nci. nih.gov/LDlink/) [9-11].

\section{Results}

\section{Assessment of 23andMe Pharmacogenetic tests}

23andMe provides pharmacogenetic reports for response to 12 drugs, which are listed in Table 1, together with the specific variants tested, the implicated gene, the phenotype tested for (adverse drug reaction (ADR), efficacy, dosage adjustment), and the FDA recommendation. The reports are from diverse clinical areas and drugs. Five reports are for variants in the cytochrome P450 genes CYP2C19 and CYP2C9, which affect both drug efficacy and adverse events. Genotypes are classified by metaboliser level and CPIC guidelines for dose adjustment or recommendations for a different drug are given. The remaining seven reports are for noncytochrome P450 drug toxicity and adverse events, such 
Table 1 Pharmacogenetic tests provided by 23andMe

\begin{tabular}{|c|c|c|c|c|c|c|}
\hline Drugs & Gene & Variant & SNP & Purpose of test & $\begin{array}{l}\text { FDA/PharmGKB } \\
\text { Guidance }^{\mathrm{a}}\end{array}$ & Guidelines $^{\mathrm{b}}$ \\
\hline Abacavir & $H L A-B$ & *57:01 & rs2395029 & Hypersensitivity reactions & $\begin{array}{l}\text { Genetic testing required } \\
\text { CPIC, DPWG }\end{array}$ & CPIC, DPWG \\
\hline Acetaldehyde & $A L D H 2$ & *2 & rs671 & $\begin{array}{l}\text { Toxicity and risk of oesophageal squamous cell } \\
\text { carcinoma }\end{array}$ & No recommendation & - \\
\hline \multirow[t]{5}{*}{ Clopidogrel (Plavix $\left.{ }^{\oplus}\right)$} & CYP2C19 & *2 & rs4244285 & Efficacy and ADRs & $\begin{array}{l}\text { Genetic } \\
\text { testing recommendation }\end{array}$ & CPIC, DPWG \\
\hline & & *3 & rs4986893 & & & \\
\hline & & *4 & rs28399504 & & & \\
\hline & & *8 & rs41291556 & & & \\
\hline & & *17 & rs12248560 & & & \\
\hline Fluorouracil (Adrucil ${ }^{\oplus}$ ) & DPYD & $* 2 \mathrm{~A}$ & rs3918290 & Toxicity and ADRs & Actionable PGx & CPIC, DPWG \\
\hline $\begin{array}{l}\text { Peginterferon alpha } \\
\text { (PEG-IFN-alpha) \& } \\
\text { ribavirin (RBV) }\end{array}$ & $\begin{array}{l}19 q 13 \\
\text { region }\end{array}$ & & rs8099917 & Hepatitis C treatment failure & Actionable PGx & CPIC \\
\hline \multirow[t]{2}{*}{ Phenytoin } & СУР2С9 & *2 & rs1799853 & Sensitivity and Dosage adjustment & Actionable PGx & CPIC, DPWG \\
\hline & & *3 & rs1057910 & & & \\
\hline \multirow{5}{*}{$\begin{array}{l}\text { Proton Pump Inhibitor } \\
\text { (PPI) }\end{array}$} & CYP2C19 & *2 & rs4244285 & Efficacy and Dosage adjustment & Informative PGX & DPWG \\
\hline & & *3 & rs4986893 & & & \\
\hline & & *4 & rs28399504 & & & \\
\hline & & *8 & rs41291556 & & & \\
\hline & & *17 & rs12248560 & & & \\
\hline Pseudocholinesterase & $B C H E$ & F1 & rs28933389 & ADR - extended paralysis and apnoea & Actionable PGx & - \\
\hline \multirow[t]{2}{*}{ Deficiency } & & $\mathrm{F} 2$ & rs28933390 & & & \\
\hline & & A & rs1799807 & & & \\
\hline Simvastatin & SLCO1B1 & *5 & rs4149056 & ADR - Myopathy & No recommendation & CPIC \\
\hline \multirow[t]{2}{*}{ Sulfonylurea } & CYP2C9 & *2 & rs1799853 & Efficacy and Dosage adjustment & No recommendation & DPWG \\
\hline & & *3 & rs1057910 & & & \\
\hline \multirow[t]{3}{*}{$\begin{array}{l}\text { Thiopurine } \\
\text { Methyltransferase }\end{array}$} & TPMT & $\begin{array}{l}\text { c. } 460 \mathrm{G}>\mathrm{A}, \\
* 3 \mathrm{~B}\end{array}$ & rs1800460 & Toxicity and ADRs & $\begin{array}{l}\text { Genetic } \\
\text { testing recommended }\end{array}$ & CPIC, DPWG \\
\hline & & $\begin{array}{l}\text { C. } 238 \mathrm{G}>\mathrm{C} \\
* 2\end{array}$ & rs1800462 & & & \\
\hline & & $\begin{array}{l}\text { c. } 719 A>G, \\
* 3 C\end{array}$ & rs1142345 & & & \\
\hline \multirow[t]{3}{*}{ Warfarin } & СYР2С9 & *2 & rs1799853 & Efficacy and Dosage adjustment & Actionable PGx & CPIC \\
\hline & & *3 & rs1057910 & & & \\
\hline & VKORC1 & & rs9923231 & & & \\
\hline
\end{tabular}

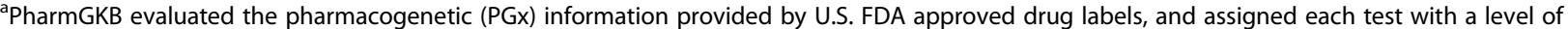
recommendation according to the level of clinical evidence, [7] The levels begin from genetic testing required, genetic test recommended, Actionable PGx to Informative PGx.

b: Guidelines from https://www.pharmgkb.org/view/dosing-guidelines.do published by the Clinical Pharmacogenetics Implementation Consortium (CPIC) or the Royal Dutch Association for the Advancement of Pharmacy - Pharmacogenetics Working Group (DPWG)

*used to indicate that these values represent a given variant in the literature

as abacavir hypersensitivity reactions which are associated with $H L A-B * 57: 01$. All reports are for variants in a single gene, except for warfarin which combines genotypes from three SNPs in CYP2C9 and VKORC1. The report for acetaldehyde toxicity, leading to increased risk of oesophageal squamous cell carcinoma in people of East Asian ancestry, is a curious entry in the list, since the toxicity arises from a breakdown of alcohol, a recreational drug, and not a pharmaceutical compound as in the other 11 tests.

The FDA considers that pharmacogenetic tests for five of the 12 drugs provide actionable results (fluorouracil, peginterferon alpha, phenytoin, pseuodocholinesterase deficiency and warfarin); genetic testing is required for 
one (abacavir); recommended in two (clopidogrel and thiopurine methyltransferase); one provides informative results (proton pump inhibitor) and the remaining three tests have no FDA recommendations (acetaldehyde, simvastatin, sulfonylurea).

For most drugs, 23andMe tests the variants listed by the FDA, but some differences exist. For phenytoin, 23andMe reports on drug sensitivity from CYP2C9*2 and *3 variants, but the U.S. FDA additionally lists pharmacogenetic information on $H L A-B^{*} 15: 02$, which confers increased risk of life threatening hypersensivity reactions like Stevens-Johnson syndrome (SJS) and toxic epidermal necrolysis (TEN) [12]. CPIC guidelines strongly recommend alternative treatments for patients with $H L A-B * 15: 02$ positive status, [13] but 23andMe does not report on $H L A-B * 15: 02$.

\section{Differences between ethnicities}

Many pharmacogenetic variants have frequencies that vary substantially by ethnicity, and different patterns of $\mathrm{LD}$ in associated regions, meaning that the relevance and interpretation of tests is not homogeneous across populations. For example, common genetic polymorphisms in $C Y P 2 C 19$ and $C Y P 2 C 9$ alter ability to metabolise drugs [14]. Individuals with $C Y P 2 C 19 * 2, * 3$, *4, *8 and $C Y P 2 C 9 * 2$, *3 polymorphisms may be poor metabolisers and may require dose reductions or an alternative drug. The frequency of CYP2C19 poor metaboliser genotypes is highest in individuals of East Asian ancestry (14\%), and is much lower in those of African ancestry (4\%) or European ancestry (2\%) [15]. Conversely, CYP2C9*2 and *3 variants are common in European populations, with frequencies of approximately $14 \%$ and $8 \%$ respectively. These variants have lower frequency in African and East Asian populations [13].

Pharmacogenetic testing for abacavir hypersensitivity is well-established, with the drug label stating "All patients should be screened for the HLA-B*57:01 allele prior to initiating therapy" [16]. Two large studies, PREDICT-1 and SHAPE, have assessed the clinical utility of this test (Table 1) $[17,18]$. The studies confirmed strong association between $H L A-B * 57: 01$ carrier status and immunologically confirmed hypersensitivity reactions, with sensitivity of $100 \%$ and specificity of $>96 \%$ in both European and African ancestries. 23andMe reports test results based on SNP rs2395029, which is in strong linkage disequilibrium with $H L A-B * 57: 01$. However, rare recombination events occur in some populations [19] and there is incomplete LD between rs2395029 and HLA-B*57:01: [20-22] 23andMe report rs2395029 heterozygotes as having a 94\% chance of carrying $H L A-B * 57: 01$. In addition, little is known about the predictive value of the SNP-based test in non-European populations. The rs2395029-G allele is extremely rare in the African population $(\sim 0 \%)$, and is only present in $1 \%$ of the East Asian population. LD between $H L A-B * 57: 01$ and rs2395029 varies by population, with perfect LD in Han Chinese and Tamil Indians, but lower in Southeast Asian Malays $\left(r^{2}=0.75\right)$. These SNP test results should therefore be interpreted with caution, particularly in non-European populations.

In peginterferon alpha (PEG-IFN-alpha) and ribavirin (RBV) combined therapy for the chronic infection of hepatitis C virus (HCV), $25-40 \%$ of patients fail to respond. Genome-wide association studies identified a significant association of rs8099917 with treatment response, with the $\mathrm{G}$ allele increasing risk of failure to response. Carriers of the rs8099917 TT genotype have a 2-3 fold increased chance of responding to therapy [23]. A second SNP rs12979860, not reported by 23andMe, may have better predictive value in some populations [24]. 23andMe reports only rs8099917, stating that "because all of these SNPs are so closely linked to each other, they are all probably representing the same effect" [25]. However, the allele frequencies and linkage disequilibrium between these variants differ across populations (Table 2), so while these SNPs are in strong LD in East Asian populations, the LD is much weaker in African populations. The validity of this test may therefore depend on ethnicity of the 23andMe customer, although it is likely that neither SNP is the causal variant.

Frequencies of the rs9923231 $\mathrm{T}$ allele, which increases sensitivity to Warfarin, vary across ethnicities. In 1000 Genomes phase 3 data, [9] the frequency is highest in Europeans (39\%), and the T allele is much rarer in South Asians (15\%), East Asians (12\%), and Africans (5\%).

For acetaldehyde toxicity, the $A L D H 2 * 2($ rs671, G > A) variant is mainly found in East Asians and is rare in other ancestries.

For 5-FU toxicity, the frequency of $D P Y D * 2 A$ is low in all populations. It is almost non-existent in AfricanAmerican and Japanese populations, and has frequency of $0.91 \%$ and $0.47 \%$ in Dutch and German populations respectively [26].

For Simvastatin-induced myopathy, the frequency of the minor allele of rs4149056 is highest in European and Asian ancestries, with very low frequencies in African populations [27]. 23andMe genotyping captures four of the five non-functional alleles which reduce TPMT

Table 2 Population genetics of SNPs rs8099917 and rs12979860, both associated with response to hepatitis $C$ treatment

\begin{tabular}{lllll}
\hline Population & rs8099917 T/G MAF G & rs12979860 C/T MAF T & $D^{\prime}$ & $r^{2}$ \\
\hline European & 0.17 & 0.31 & 0.97 & 0.43 \\
East Asian & 0.08 & 0.08 & 0.99 & 0.92 \\
African & 0.04 & 0.67 & 1.00 & 0.02
\end{tabular}

Minor allele frequencies (MAF) calculated using 1000 Genomes data; LD parameters calculated using LDlink $[9,10]$ 
activity. The frequency of the functional "1 allele ranges from 0.925 in Mexicans to 0.983 in Asians ${ }^{18}$ with frequencies of the non-functional markers also varying by ethnicity. The other non-functional alleles are not tested for by $23 a n d M e$, but have a frequency of $<0.1 \%$ in all populations tested [28].

\section{Limitations of 23andMe pharmacogenetic tests}

For a number of the pharmacogenetic tests performed by $23 \mathrm{andMe}$, the most recent literature has additional information on associations, making 23andMe's tests incomplete in their assessment of ADRs or efficacy.

For 5-FU, 23andMe tests only for the $2 A$ variant, but as 23andMe state on their website, further variants in the gene are associated with 5-FU toxicity. For example, recent studies have shown that $D P Y D^{*} 13$, rs67376798, c.1679 $\mathrm{T}>\mathrm{G}$ and c.1236G > A/HapB3 are also associated with 5-FU toxicity [28-30].

For pseudocholinesterase deficiency, 23andMe provides reports on three variants, fluorine resistant 1 (F1), fluorine resistant 2 (F2) and dibucaine resistant mutation (A). Other mutations in $B C H E$ with similar effect are not reported by 23andMe, such as K-variant, silent-1, silent-2 and silent-7 [31]. The protocol for selecting the specific variants to be tested by 23andMe is not described and only two citations are listed for this particular drug response, and which does not meet their selection criteria [4].

For acetaldehyde toxicity, 23andMe reports on rs671, which is mainly present in East Asians. An additional variant rs1229984, $\mathrm{A}>\mathrm{G}$ in $A D H 1 B$, increases risk of oesophageal squamous cell carcinoma and is present in multi-ethnic populations. Cui R et al. (2009) found that the combination of $A L D H 2 * 2$ and $A D H 1 B$ variants with smoking and drinking significantly increases the risk of oesophageal squamous cell cancer 189-fold [32].

Assessing variation in TPMT is difficult and 23andMe states that their current technology cannot distinguish between the genetic changes in the same or different copies of the TPMT gene. For example, it is not possible to differentiate between one copy of * $3 A$ (reduced function) and copies of $* 3 B$ and $* 3 C$ on different chromosomes (no function). Although it is rare to carry both $* 3 B$ and $* 3 C$ variants, incorrect interpretation of test results could lead to dosing errors.

\section{Discussion}

Adverse events from drug hypersensitivity currently place a large burden on healthcare costs and preventable adverse drug events could cost the UK NHS up to $£ 2.5$ billion per year [33]. Some of these events are associated with genetic factors, and so may be preventable through pharmacogenetic testing. DTC pharmacogenetic testing may have a role to play in either raising awareness of pharmacogenetic testing, or providing customers with preliminary information, which could be followed up with healthcare professionals. However, these benefits need to be balanced with the risks involved in providing medical information that is not communicated through a healthcare professional.

The reports provided by 23andMe to UK customers highlight the challenges of pharmacogenetic testing, some of which are unique to a SNP-based genotyping platform as used by the DTC company, others of which are applicable to any technology, and any method of delivery. We compared the 12 reports provided by 23andMe with recommendations from the FDA and CPIC, finding several differences between the two sources. 23andMe report on some pharmacogenetic tests for which FDA provided no recommendation (simvastatin, sulfonylurea and acetaldehyde); these tests are supported by research publications and therefore (mostly) meet the selection criteria specified by 23andMe. Not all pharmacogenetic tests with FDA recommendations or CPIC guidelines are reported by 23andMe. Some omissions may be due to difficulties in tagging the relevant pharmacogenetic variant. For example, 21\% (44/204) of the tests in the FDA Table of Pharmacogenomic Biomarkers in Drug Labelling are for CYP2D6 alleles. These cannot be assessed fully with a genome-wide genotyping chip due to gene deletions and sequence similarity with pseudogenes, and this may explain the lack of CYP2D6 tests in 23andMe's portfolio. Some tests provided by 23 andMe provide an incomplete assessment of risk variants within the target genes (DPYD, ADH1B). Similarly, for phenytoin, 23andMe assesses CYP2C9 variation, while the FDA's table lists tests for CYP2C9, CYP2C19 and HLA-B*15:02, with CPIC providing guidelines for joint tests across $C Y P 2 C 9$ and $H L A-B * 15: 02$. This omission is potentially serious since $H L A-B * 15: 02$ carriers are at increased risk of SJS/PTEN, and use of phenytoin in not recommended. Similarly, carbamazepine-induced SJS/PTEN occurs in HLA-B*15:02 carriers. This variant is not reported by 23andMe, although it is particularly relevant in East Asians where $H L A-B * 15: 02$ frequency is highest. Two SNPs in strong LD with HLA$B * 15: 02$ have been identified. A sample of 45 Han Chinese from Beijing, China showed $r^{2}=1$ with rs3909184 and rs2844682, [34] but LD with rs3909184 was much weaker in an Asian Pacific Islander population (sensitivity 32\%; no $\mathrm{r}^{2}$ value given). These population-level differences in LD between HLA alleles and tagging SNPs make it difficult to implement a pharmacogenetic test using only SNP data from genotyping arrays, particularly in a DTC setting which does not allow for subtleties in test interpretation. This highlights the point that fully comprehensive pharmacogenetic testing would require a combination of SNP genotyping or sequencing, plus HLA serotyping.

For drug metabolising enzymes, 23andMe provide reports on $\mathrm{CYP} 2 \mathrm{C} 19$ for clopidogrel and proton pump 
inhibitors, but not for other drugs such as the widely prescribed selective serotonin reuptake inhibitor antidepressants (SSRIs). CYP2C19 tests are listed on the FDA table for SSRIs, and are available in a commercial test by AssureX [35]. Guidelines for CYP2C19 pharmacogenetic tests are provided by CPIC ( 8 drugs) and the Dutch Working Group (12 drugs), each of which includes the test provided by 23andMe.

One of the challenges in pharmacogenetics is the diverse allele frequencies across populations. Different allele frequencies may make the test only relevant in a single population, as in testing for phenytoin and $H L A-B * 15: 02$ which is mainly found in Asian populations. Differing allele frequencies for the causal variant does not affect interpretation of the pharmacogenetic test result, but different linkage disequilibrium may invalidate interpretation of a tagging SNP, as seen with rs8099917 and Hepatitis-C treatment response. Without further work refining causal SNPs at such loci, it is probable that pharmacogenetic tests will provide different degrees of sensitivity across ethnicities.

We have focused on DTC company, 23andMe, since they are the largest provider in this market, and are commendably open about the exact SNPs genotyped, and the reports generated for each pharmacogenetic test - therefore enabling scrutiny of the service offered. These pharmacogenetic reports are available to 23andMe customers in the UK, Canada and some EU countries, but are not currently provided to US customers. A 2012 review of DTC pharmacogenetic testing companies, identified eight companies that provided at least one pharmacogenetic test [1]. Some of the companies surveyed require tests to be ordered through a clinican (Genelex, Pathway genomics), or are no longer in the market (Navigenics). Geneplanet now provides DTC pharmacogenetics for six drugs or drug classes (clopidogrel, metformin, omeprazole, perindopril, statins, warfarin), and Theranostics for two drugs (clopidogrel, statins) but neither company gives detailed information on the tests or reported outcomes and so are not included here.

DTC pharmacogenetic testing has the potential to benefit patients by educating individuals about genetics and providing preliminary information that patients could follow up with a medical professional. 23andMe's public website and customer reports make clear that they do not provide a medical service and that the report is not a diagnostic test. Reports give risks (\%) of an adverse event, and general comments such as 'may benefit from a different dose'; they instruct customers to "consult with a healthcare provider about ... appropriate next steps", and not to "make any changes to any current treatment without first consulting a healthcare provider". For each report, 23andMe provides detailed information about the test performed, the evidence for gene-drug association, and the implications of test results. In addition to clinicalrelated information, full SNP genotypes are returned. Pharmacogenetics is a particularly relevant focus for DTC testing since the test has value throughout life, and need be performed only once. This makes implementation of a pre-emptive pharmacogenetic testing panel potentially cost-effective, since the results can be interrogated when any new drug is prescribed.

1. The service offered by 23andMe has several important limitations, and for the service offered to be more effective, we would recommend a number of changes, such as better consideration and documentation of differences across ancestry. For example, the literature shows substantial differences in sensitivity and specificity of tests for Hepatitis- $\mathrm{C}$ treatment response between ancestries. This should be fully reported by 23andMe to ensure all test users understand the ancestry-specific limitations of testing. In the absence of research linking the tested SNP to the response in a given consumer's ethnicity, this limitation should be communicated clearly.

2. Better mechanisms should be in place to ensure that tests reflect the latest science. Pharmacogenetic research can move quickly, and producing out-ofdate or incomplete reports raises ethical questions.

3. More consideration of what tests should be reported. Many 23andMe customers will not be knowledgeable about the relative importance of the different tests, and reporting tests that are not supported by the FDA or other guidelines could cause unnecessary concern or anxiety for customers.

Sequencing technologies may be required to achieve these aims, as the current genotyping arrays do not always offer the variants required to perform effective testing, and would enable a wider range of important drug metabolising enzyme variation to be detected [36]. In addition, rare variants not identified in the literature will continue to provide challenges to pharmacogenetic testing on a large scale, and reduce the specificity of tests performed.

\section{Conclusions}

Pharmacogenetic testing is likely to become a routine part of clinical practice in the future. As it stands, the DTC service offered by 23andMe has limitations but has a role to play in familiarising people with genetics and raising awareness. In particular, there are challenges in providing a service across all ancestry groups when research to date has not fully resolved the causal variants at associated loci. 


\section{Abbreviations}

CPIC: Clinical Pharmacogenetics Implementation Consortium; DPWG: Dutch Pharmacogenetics Working Group; DTC: Direct-to-consumer; FDA: United States Food \& Drug Administration; LD: Linkage disequilibrium

\section{Acknowledgements}

None.

\section{Funding}

This paper represents independent research funded by the National Institute for Health Research (NIHR) Biomedical Research Centre at South London and Maudsley NHS Foundation Trust and King's College London and by the NIHR Biomedical Research Centre at Guy's and St Thomas' NHS Foundation Trust and King's College London. The views expressed are those of the author(s) and not necessarily those of the NHS, the NIHR or the Department of Health.

\section{Availability of data and materials}

All information used in this study is available from the 23andMe website which can be accessed at https://www.23andme.com/en-gb/health/reports.

\section{Authors' contributions}

ML, CML, MT performed the analysis. CML conceived of the study and participated in its design and coordination. All authors read and approved the final manuscript.

\section{Competing interests}

The authors declare that they have no competing interests.

\section{Consent for publication}

Not applicable.

\section{Ethics approval and consent to participate}

As the research reported herein does not deal directly with sensitive data ethics approval and was not required and consent was not sought. This is in line with UK ethics guidelines available at https://www.gov.uk/government/ publications/health-research-ethics-committees-governance-arrangements.

\section{Publisher's Note}

Springer Nature remains neutral with regard to jurisdictional claims in published maps and institutional affiliations.

\section{Author details}

'Department of Medical and Molecular Genetics, King's College London, 8th Floor Tower Wing, Guy's Hospital, Great Maze Pond, London SE1 9RT, UK ${ }^{2}$ SGDP Centre, Institute of Psychiatry, Psychology \& Neuroscience, King's College London, De Crespigny Park, Denmark Hill, London SE5 8EF, UK.

Received: 3 January 2017 Accepted: 28 May 2017

Published online: 19 June 2017

\section{References}

1. Chua EW, Kennedy MA. Current state and future prospects of direct-toconsumer Pharmacogenetics. Front Pharmacol. 2012;3:152.

2. Rasmussen-Torvik LJ, Stallings SC, Gordon AS, Almoguera B, Basford MA, Bielinski SJ, et al. Design and anticipated outcomes of the eMERGE-PGX project: a multicenter pilot for preemptive pharmacogenomics in electronic health record systems. Clin Pharmacol Ther. 2014;96:482-9.

3. Burton A. Are we ready for direct-to-consumer genetic testing? Lancet Neurol. 2015;14:138-9.

4. Dandekar S, Chang E, Hromatka B, Chubb A, Wu S. Guidelines on Vetting and Reporting Variants with Strong Effects on Health [PDF]. 23andMe; 2014. https://23andme.https.internapcdn.net/res/pdf/45NSStEUhM8G-e_5JXdTUw 23-07_Vetting_Variants.pdf. Accessed 9 Dec 2016.

5. Table of Pharmacogenomic Biomarkers in Drug Labeling: U.S. Food and Drug Administration; 2015. http://www.fda.gov/drugs/scienceresearch/ researchareas/pharmacogenetics/ucm083378.htm. Accessed 8 Dec 2016

6. Relling M, Klein T. CPIC: clinical Pharmacogenetics implementation Consortium of the Pharmacogenomics research network. Nature. 2011;89:464-7.

7. Drug Labels: PharmGKB; 2016. https://www.pharmgkb.org/view/drug-labels. do. Accessed 8 Dec 2016
8. Swen J, Nijenhuis M, Boer AD, Grandia L, AM-vd Z, Mulder H, et al Pharmacogenetics: from bench to byte- an update of quidelines. Clin Pharmacol Ther. 2011;89:662-73.

9. Auton A, Brooks LD, Durbin RM, Garrison EP, Kang HM, Korbel JO, et al. A global reference for human genetic variation. Nature. 2015;526:68-74.

10. Machiela MJ, Chanock SJ. LDlink: a web-based application for exploring population-specific haplotype structure and linking correlated alleles of possible functional variants. Bioinformatics. 2015;31:3555-7.

11. Johnson AD, Handsaker RE, Pulit SL, Nizzari MM, O'Donnell CJ, de Bakker PI. SNAP: a web-based tool for identification and annotation of proxy SNPS using HapMap. Bioinformatics. 2008;24:2938-9.

12. Dilantin ${ }^{\circledast}$ (extended phenytoin sodium capsules, USP): Parke-Davis Div of Pfizer Inc; 1976. http://www.accessdata.fda.gov/drugsatfda_docs/label/2016/ 008762s054,010151s041lbl.pdf. Accessed 8 Dec 2016.

13. Caudle KE, Rettie AE, Whirl-Carrillo M, Smith LH, Mintzer S, Lee MTM, et al. Clinical Pharmacogenetics implementation Consortium guidelines for CYP2C9 and HLA-B genotypes and Phenytoin dosing. Clin Pharmacol Ther. 2014;96:542-8

14. Ogu CC, Maxa JL. Drug interactions due to cytochrome P450. BUMC Proceedings. 2000;13:421-3.

15. Gould DB, Phalan FC, van Mil SE, Sundberg JP, Vahedi K, Massin P, et al Role of COL4A1 in small-vessel disease and hemorrhagic stroke. N Engl J med. 2006:354:1489-96.

16. Abacavir Label. 2015. http://www.accessdata.fda.gov/drugsatfda_docs/label/ 2015/020977s030,020978s034lbl.pdf. Accessed 8 Dec 2016.

17. Saag M, Balu R, Phillips E, Brachman P, Martorell C, Burman W, et al. High sensitivity of human leukocyte antigen-b*5701 as a marker for immunologically confirmed abacavir hypersensitivity in white and black patients. Clin Infect Dis. 2008;46:1111-8.

18. Mallal S, Phillips E, Carosi G, Molina JM, Workman C, Tomazic J, et al. HLA-B*5701 screening for hypersensitivity to abacavir. N Engl J med. 2008:358:568-79.

19. Martin MA, Hoffman JM, Freimuth RR, Klein TE, Dong BJ, Pirmohamed M, et al. Clinical Pharmacogenetics implementation Consortium (CPIC) guidelines for HLA-B genotype and Abacavir dosing: 2014 update. Clin Pharmacol Ther. 2014:95:499-500.

20. Badulli C, Sestini R, Sbarsi I, Baroncelli M, Pizzochero C, Martinetti M, et al. Tag SNPs of the ancestral haplotype 57.1 do not substitute HLA-B*57:01 typing for eligibility to abacavir treatment in the Italian population. Pharmacogenomics. 2012:13:247-9.

21. Rodriguez-Novoa S, Cuenca L, Morello J, Cordoba M, Blanco F, JimenezNacher I, et al. Use of the HCP5 single nucleotide polymorphism to predict hypersensitivity reactions to abacavir: correlation with HLA-B*5701. J Antimicrob Chemother. 2010;65:1567-9.

22. Melis R, Lewis T, Millson A, Lyon E, McMillin GA, Slev PR, et al. Copy number variation and incomplete linkage disequilibrium interfere with the HCP5 genotyping assay for abacavir hypersensitivity. Genet Test Mol Biomarkers. 2012;16:1111-4.

23. Hayes CN, Imamura M, Aikata H, Chayama K. Genetics of IL28B and HCV-response to infection and treatment. Nat rev Gastroenterol Hepatol. 2012;9:406-17.

24. Ge D, Fellay J, Thompson AJ, Simon JS, Shianna KV, Urban TJ, et al. Genetic variation in IL28B predicts hepatitis C treatment-induced viral clearance. Nature. 2009;461:399-401.

25. SNPwatch: More Evidence That Genetic Variations are Important for Hepatitis C Infection and Treatment. 23andMe. 2009. https://blog.23andme.com/news/ snpwatch-more-evidence-that-genetic-variations-are-important-for-hepatitis-cinfection-and-treatment/ - eh3jgCMb5xEkjrQm.99. Accessed 8 Dec 2016.

26. $A B P \vee-K$. Dihydropyrimidine dehydrogenase and the efficacy and toxicity of 5-fluorouracil. Eur J Cancer. 2004:40:939-50.

27. Ramsey LB, Johnson SG, Caudle KE, Haidar CE, Voora D, Wilke RA, et al. The clinical Pharmacogenetics implementation Consortium guideline for SLCO1B1 and Simvastatin-induced Myopathy: 2014 update. Clin Pharmacol Ther. 2014;96:423-8.

28. Amstutz U, Froehlich TK, Largiader CR. Dihydropyrimidine dehydrogenase gene as a major predictor of severe 5-fluorouracil toxicity. Pharmacogenomics. 2011;12:1321-36

29. Morel A, Boisdron-Celle M, Fey L, Soulie P, Craipeau MC, Traore S, et al. Clinical relevance of different dihydropyrimidine dehydrogenase gene single nucleotide polymorphisms on 5-fluorouracil tolerance. Mol Cancer Ther. 2006;5:2895-904 
30. Meulendijks D, Henricks LM, Sonke GS, Deenen MJ, Froehlich TK, Amstutz U, et al. Clinical relevance of DPYD variants c.1679T>G, c.1236G >a/HapB3, and c.1601G $>$ a as predictors of severe fluoropyrimidine-associated toxicity: a systematic review and meta-analysis of individual patient data. Lancet Oncol. 2015;16:1639-50.

31. Yen T, Nightingale BN, Burns JC, Sullivan DR, Stewart PM.

Butyrylcholinesterase (BCHE) genotyping for post-Succinylcholine apnea in an Australian population. Clin Chem. 2003;49:1297-308.

32. Cui R, Kamatani Y, Takahashi A, Usami M, Hosono N, Kawaguchi T, et al. Functional variants in ADH1B and ALDH2 coupled with alcohol and smoking synergistically enhance esophageal cancer risk. Gastroenterology. 2009;137:1768-75.

33. Exploring the costs of unsafe care in the NHS London: Frontier Economics Ltd; 2014. http://www.frontier-economics.com/documents/2014/10/ exploring-the-costs-of-unsafe-care-in-the-nhs-frontier-report-2-2-2-2.pdf. Accessed 8 Dec 2016.

34. de Bakker PI, McVean G, Sabeti PC, Miretti MM, Green T, Marchini J, et al. A high-resolution HLA and SNP haplotype map for disease association studies in the extended human MHC. Nat Genet. 2006;38:1166-72.

35. Altar CA, Carhart JM, Allen JD, Hall-Flavin DK, Dechairo BM, Winner JG. Clinical validity: combinatorial pharmacogenomics predicts antidepressant responses and healthcare utilizations better than single gene phenotypes. Pharmacogenomics J. 2015;15:443-51.

36. Numanagic I, Malikic S, Pratt VM, Skaar TC, Flockhart DA, Sahinalp SC. Cypiripi: exact genotyping of CYP2D6 using high-throughput sequencing data. Bioinformatics. 2015;31:i27-34.

\section{Submit your next manuscript to BioMed Central and we will help you at every step:}

- We accept pre-submission inquiries

- Our selector tool helps you to find the most relevant journal

- We provide round the clock customer support

- Convenient online submission

- Thorough peer review

- Inclusion in PubMed and all major indexing services

- Maximum visibility for your research

Submit your manuscript at www.biomedcentral.com/submit

) Biomed Central 